therapeuten getragen werden müssen. Ob diese Investition eventuell 2013 durch ein höheres Honorar kompensiert werden kann, ist völlig offen.

Angesichts einer solchen Entwicklung ist es nicht verwunderlich, dass zwei Drittel der Ärzte laut einer Studie des Meinungsforschungsinstituts Allensbach, des Finanzdienstleisters MLP und der Bundesärztekammer befürchten, dass in der Zukunft neben therapeutischen Gesichts- punkten immer stärker wirtschaftliche Aspekte bei der Versorgung der Patienten eine Rolle spielen werden. Mehr als $72 \%$ der Ärzte sehen wegen des Kostendrucks schon heute ihre Therapiefreiheit bedroht oder zumindest infrage gestellt. Die Hälfte der befragten Ärzte konstatiert, dass der Arztberuf an Attraktivität verloren habe.

Viele von ihnen hoffen nicht mehr auf ein Umdenken. Sie können es sich kaum mehr vorstellen, dass sich an den Arbeitsbedingungen etwas ändert. Im Gegenteil, mehr als die Hälfte der Mediziner befürchtet, dass die Attraktivität des Arztberufes weiter abnehmen könnte. Welche Auswirkungen ein solches Stimmungsbild auf die nachrückende Generation und den jetzt schon vorhandenen Ärztemangel haben wird, bedarf sicherlich keiner weiteren Erörterung.

\title{
Update: So können Einträge in Bonushefte berechnet werden
}

- Ärztliche Eintragungen in GKV-Bonushefte gehören eindeutig nicht zur vertragsärztlichen Versorgung.

Das Bonusheft in der GKV wurde bisher lediglich als Vorsorgenachweis bei der zahnärztlichen Versorgung eingeführt. Das ignorieren die Krankenkassen und haben darüber hinaus mit dem Bonusheft einhergehende Bonifikationen für ihre Versicherten definiert. Denen wird zum Beispiel ein Bonus gewährt, wenn sie regelmäßig gesetzliche Früherkennungsuntersuchungen oder qualitätsgesicherte Leistungen der Krankenkassen zur Primärprävention in Anspruch nehmen. Zu diesem Zweck händigen die Krankenkassen ihren Versicherten Bonus- hefte aus, in denen der Arzt die Teilnahme an einer solchen Früherkennungsuntersuchung mit Stempel und Unterschrift bestätigen soll.

Diese Zuwendungen sind jedoch freiwillige Leistungen der Krankenkassen und die dazu gehörigen ärztlichen Eintragungen in Bonushefte gehören nicht zur vertragsärztlichen Versorgung. Daher steht hierfür auch keine EBM-Position zur Verfügung. Die Leistung kann folgerichtig privat und in der Regel nach Nr. 70 GOÄ abgerechnet werden.

\section{MMW Kommentar}

Wichtig ist, dass vor einer solchen Liquidation das Einvernehmen mit dem Patienten hergestellt wird. Formalrechtlich ist sogar eine Schriftform erforderlich. Bewusst versuchen viele Kassen wegen dieser einzuhaltenden Formalien gerne einen Keil zwischen Arzt und Patient zu treiben. Dies sollte auf keinen Fall Anlass sein, die Eintragung kostenlos vorzunehmen. Alternativ kann sich der Patient nach der Rechtsprechung und der Berufsordnung eine Kopie der Behandlungsunterlagen gegen Kostenerstattung in Höhe von 50 Cent pro DIN A4-Seite aushändigen lassen und dies als Nachweis gegenüber der Kasse verwenden, um in den Genuss der Bonusleistung zu gelangen. In diesem Fall muss die Kasse die Eintragungen ins Bonusheft vornehmen.

\section{Kostenlose Behandlung verboten}

- Nach einem aktuellen Urteil des Landgerichts Berlin (LG) darf ein Arzt seine Patienten nicht kostenlos behandeln, da eine solche Maßnahme gegen geltendes Wettbewerbsrecht verstößt (Az. $103 \mathrm{O}$ $80 / 20)$. Im vorliegenden Fall hatte ein Arzt kostenlose Vorsorgeuntersuchungen angeboten. Das LG Berlin sah in dem Angebot des Arztes einen Verstoß gegen $\$ 12$ der Berufsordnung. Dieser schreibt vor, dass die ärztliche Honorarforderung angemessen sein muss. Für die Bemessung sei die Amtliche Gebührenordnung (GOÄ) Grundlage, soweit nicht andere gesetzliche Vergütungsregelungen gelten. Demnach dürften Ärzte die Sätze nach der GOÄ nicht in unlauterer Weise unterschreiten oder gar aufheben. Die Kammer verbot dem Arzt deshalb, die Leistungen kostenlos anzubieten.

\section{MMW Kommentar}

Ärztliche Leistungen dürfen nach den strengen Grundsätzen der Berufsordnung weder kostenlos noch zu günstigen Konditionen angeboten werden. Solche Aktionen verstoßen dabei nicht nur gegen die Berufsordnung, sondern auch gegen das Wettbewerbsrecht. In allen Fällen gilt aber selbstverständlich: Wo kein Kläger ist, gibt es auch keinen Richter! 